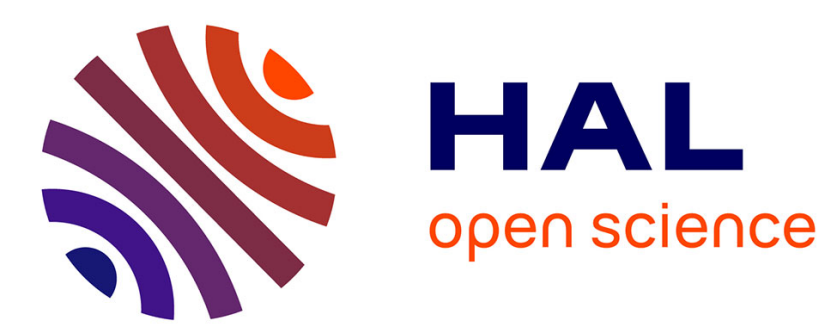

\title{
Elements of qualitative cognition: An information topology perspective
}

Pierre Baudot

\section{To cite this version:}

Pierre Baudot. Elements of qualitative cognition: An information topology perspective. Physics of Life Reviews, 2019, pp.263-275. 10.1016/j.plrev.2019.10.003 . hal-02457799

\section{HAL Id: hal-02457799 \\ https://hal-amu.archives-ouvertes.fr/hal-02457799}

Submitted on 28 Jan 2020

HAL is a multi-disciplinary open access archive for the deposit and dissemination of scientific research documents, whether they are published or not. The documents may come from teaching and research institutions in France or abroad, or from public or private research centers.
L'archive ouverte pluridisciplinaire HAL, est destinée au dépôt et à la diffusion de documents scientifiques de niveau recherche, publiés ou non, émanant des établissements d'enseignement et de recherche français ou étrangers, des laboratoires publics ou privés. 


\title{
Review
}

\section{Elements of qualitative cognition: An information topology perspective}

\author{
Pierre Baudot ${ }^{\mathrm{a}, \mathrm{b}, *}$ \\ a Median Technologies, Valbonne, France \\ ${ }^{\mathrm{b}}$ Inserm UNIS UMR1072, Université Aix-Marseille AMU, Marseille, France
}

\begin{abstract}
Elementary quantitative and qualitative aspects of consciousness are investigated conjointly from the biology, neuroscience, physic and mathematic point of view, by the mean of a theory written with Bennequin that derives and extends information theory within algebraic topology. Information structures, that accounts for statistical dependencies within n-body interacting systems are interpreted a la Leibniz as a monadic-panpsychic framework where consciousness is information and physical, and arise from collective interactions. The electrodynamic intrinsic nature of consciousness, sustained by an analogical code, is illustrated by standard neuroscience and psychophysic results. It accounts for the diversity of the learning mechanisms, including adaptive and homeostatic processes on multiple scales, and details their expression within information theory. The axiomatization and logic of cognition are rooted in measure theory expressed within a topos intrinsic probabilistic constructive logic. Information topology provides a synthesis of the main models of consciousness (Neural Assemblies, Integrated Information, Global Neuronal Workspace, Free Energy Principle) within a formal Gestalt theory, an expression of information structures and patterns in correspondence with Galois cohomology and discrete symmetries. The methods provide new formalization of deep neural network with homologicaly imposed architecture applied to challenges in AI-machine learning.
\end{abstract}

Keywords: Qualitative cognition; Sensory coding; Algebraic topology; Information theory; Constructive logic; Thermodynamic

\section{Introduction. "Unity and diversity" [1]: the monadic view}

This review simply present, recycle and combine, in a consistent fashion, well-established results, such that the resulting theory is the least partial possible and unified. An important part of the ideas presented here are the result of a long-lasting collaboration with Bennequin [2]. In the world of ideas, nothing is lost, nothing is created, everything transforms. We will focus on an old intuitionist idea of a mathematical and physical nature of our subjective being, and even of our most elementary perceptions. A more detailed exposition can be found in [3]. An important part,

\footnotetext{
* Corresponding author at: Median Technologies, Valbonne, France.

E-mail address: pierre.baudot@mediantechnologies.com.

URL: https://sites.google.com/view/pierrebaudot/.
} 
if not all, of the results in neuroscience imaging, electrophysiological studies, psychophysic and psychology studies concerns more or less directly consciousness. The results of such studies, as advocated and centrally underlined by the Integrated Information Theory (IIT) of Tononi and Edelmann, tend to be that forms of consciousness are very diverse [1]. Neuroscience and cognitive sciences have developed specialized concepts and taxonomy for these different forms, such as attention, low-level vision, audition, multi-modal integration, decision, motor planning, short-term memory, etc. In a sense, there exists a given, particular name of consciousness for each function and associated structure in nervous systems. Moreover, there exists a wide diversity of nervous systems: human, macaque, cat, rat, mouse, zebra finch, bat, turtle, elephantfish, cricket, fly, squid, aplysia, worms (caenorhabditis elegans), to cite just a few generic experimental models. Such a diversity reveals the richness of cognitive forms [4]. Each of them have remarkably different structures and functions. The point of view adopted here, now more accepted in neuroscience [5], is that if one accepts that there exists a qualitative low-level perception in humans, and admits it provides a quite elementary form of consciousness, one should accept from the purely empirical criterion of observability that the echolocation of a bat, for example, is also associated with elementary forms of consciousness, albeit likely to be different from the one we experience, as can be inferred from electro-physiological studies and discussed by Nagel [6]. What is presented here reconciles the IIT [1,7] and the Global Workspace [8-10] models by proposing that what one may consider as an unconscious state is "someone else's" consciousness. This proposition can be simply illustrated by the development in patients, specifically called "split-brain" patients, of two quite independent consciousness streams following a callosotomy, as studied notably in the celebrated work of Sperry and Gazzaniga [11,12]. Here, we postulate the objects of our subjective experiences or perceptions exist and the existence of the subject that perceives (the "I think therefore I am" of Descartes) and complete it with a statement along the lines of "It moves therefore it is", a phenomenological definition of anima based on motion. The present view makes no fundamental distinction between reflexive and qualitative consciousness called qualia, and avoid the usual dualist point of view recalled by Chalmers [13]: a synthetic monadic or monist view that follows Leibniz's 'monads' [14,15] without ontological difference between the mind and the body. More precisely, there is a reflexive mechanism associated to any qualitative experience and there is a qualitative mechanism associated with any reflexive experience, developed here in terms of an internal and free energy respectively (cf. 3).

\section{Electrophysiology of elementary percepts: information flows}

\subsection{Consciousness: the electrodynamic view - "Where is my mind?"}

Since the work of Galvani in 1771 on "animal electricity" [16], electric and more generally electromagnetic signals have provided the main sources of observable phenomena for neuroscience and cognitive studies, and yet provide the basis of consciousness theory, at least in this review. Since Galvani, experiments have become more precise and provide empirical measurements of electromagnetic signals at many different scales, that is, with varying space-time resolutions, ranging from single molecule channels to the whole brain, as is the case in fMRI or EEG recordings. An out of date and non-exhaustive comparative figure of the space-time resolutions of some measures of the electromagnetic field given off by activity in the central nervous system is given in [17,18]. Studies into impulsional response and variability at the different organizational scales of the nervous system, from channels to cortical areas is reviewed in the associated extended version of the review [3]. The basic proposition of this review from a physical point of view is that the theory of consciousness is the theory of electromagnetic fields (leaving aside the effects of gravity). The electromagnetic theory of consciousness has been developed on the basis of the theory of oscillating neural assemblies [19-21], and basically considers the idea that the spectrum of electrical activity observable in Electroencephalograms (EEGs), typically ranging from 0 to $100 \mathrm{~Hz}$, sustains consciousness. The proposition here is to broaden the spectrum to any frequency and to take into account the temporal and phase dynamics of the activity in question. The beta $(12-40 \mathrm{~Hz})$ and gamma $(40-100 \mathrm{~Hz})$ frequencies are simply particular activity ranges evoked by conscious states in humans and in primates more generally, and are mainly generated by primates' peculiar cortical (or cortical-like, e.g. olfactory bulb) excitatory-inhibitory microcircuits. They do not account for the activity related to consciousness observed using other methods at different scales and in other species. This proposition is in fact simply an up-to-date reconsideration of the statement attributed to Pythagoras: “All things feel!". It simply proposes that there are no more fundamental mysteries in the black box of a human brain, nor any fewer, than in the black box of a particle collider or bubble chamber. Such a proposition includes non-spiking activity, for example graded potential neural activity as reviewed by Juusola [22], 
and also the activity of non-neural cells such as Glial cells, which display sensory responses although very slowly (due to their large capacitance) and even tuning, as shown by Sur et al. [23]. Such Glial activity can be conceived of as a component of consciousness, albeit a slowly-operating one. This proposition of the electromagnetic nature of consciousness does not exclude chemical reactions. Bio-cellular signaling or even metabolic chains are, from the physical point of view, biochemical instances of electromagnetism. For example, Preat and colleagues showed the involvement of intracellular signaling in Drosophila behavior and long-term memory formation [24]. Genetic expressions and regulations are also electromagnetic processes, and their impact on macroscospic electrical activity is further underlined by the fact that they are involved in the electrical phenotypes, such as phasic or tonic, of neurons, as shown by Soden and colleagues [25]. As cited by Monod, Wyman, Changeux in their work on allostery, "It is certain that all bodies whatsoever, though they have no sense, yet they have perception ... and whether a body be alterant or altered, evermore a perception preceded operation; for else all bodies would be alike to one another" (Francis Bacon, 1967, [26]). To give an example of an information-theoretic treatment of such a cellular perception, chemotaxis, the chemically guided movement of cells, can be looked at in terms of considering the mutual information between the input gradient and the spatial distribution [27]. Such a view includes plants, as action potentials occur in most if not all plants [28]. How far in the elementary organizations of matter is it possible to pursue the consideration of some elementary perception, action and consciousness? What is an electrodynamic theory of consciousness at the elementary level? Consider the simple Feynman diagram of elementary particle interaction, represented by the scattering process $X+Y \rightarrow X^{\prime}+Y^{\prime}$. As long as one only considers the empirical and observable considerations, that is, if one takes a phenomenological point of view, it is legitimate to consider that the proton $Y$ perceived the electron $X$ via the photon $Z$, with a "reaction" of the proton leading it to $Y^{\prime}$. Any signal received or propagated by our nervous system is at the elementary level in this way and mediated by boson-like particles. Psychophysical experiments can partially illustrate the establishing of such an elementary percept. Holmes showed that humans can sense light flashes containing as few as three photons [29], and Tinsley and colleagues showed that humans can detect a single-photon incident on the cornea with a probability significantly above chance [30]. Elementary auditory perception was also studied by Bialek and Schweitzer, who established that the sensitivity of ears can reach the limit dictated by the quantum uncertainty principle [31]. The conclusion of this study is that the measurement apparatus, i.e. the receptor cell, operates in a condition analogous to a 0 Kelvin ground state which maintains quantum coherence. From a more biophysical perspective, single action quanta and quantum formalism have been shown to be relevant to the model of the potassic ion channel selectivity filter that generates important macroscopic patterns of electrophysiological activity in neurons [32]. From an experimental point of view, it is clear that quantum effects are relevant to nervous system models and that attempts to model with precision should take quantum formalism into account. Bohr originally gave a cognitive and biologic view of quantum physics in his book "Atomic Physics and Human Knowledge" [33], further highlighting that quantum physics is not just a theory of physics, but also a theory of what one can objectively know about physics. Since Bohr's work, many works have proposed to examine consciousness and the nervous system on the basis of quantum entanglement and decoherence, or even quantum gravity principles, as in the celebrated works of Hameroff and Penrose [34], which proposed a specific involvement of cytoskeletal microtubules. Penrose's propositions [35] fall within the bounds of the present framework from a physical point of view, while his biological proposition involving microtubules, over-restrictive with respect to the current corpus of knowledge on the dynamics of the nervous system, is extended here to the whole nervous system's dynamic. With regard to the question, "Where is my mind?", we conclude that biological studies have reported that it can be found at all organizational scales and locations of the nervous system. As a result, the nervous system can be seen as a constantly adapting system with a range of plasticity and homeostatic processes operating at different scales of time and space [36]. By this, we mean that the substrate of memory in the nervous system can be and has been found virtually everywhere, from genetic expression, kinase and/or calcium intracellular signaling cascades, the synaptic NMDA mechanism, to neuronal morphology including synaptic formation, cortical maps of areas remodeling etc. In electrodynamics, the formalism accounting for such multi-scale dynamics is one of its core tenets: the renormalization theory [37,38], as reviewed by Shirkov [39] and Huang [40].

\subsection{Information assemblies and flows - "I, you, it, he, she, we all from the bit"}

Rate and temporal codes of consciousness, digital-spike and analog-Vm coding. The coding unit-event of the nervous system has been considered to be the spike - what has been called spike coding, a binary code. It assumes 
that spike waveform and initiation and synaptic transmission are all-or-nothing processes. The probabilistic functional approach can be used to investigate the code's temporal precision. The book by Rieke and colleagues provides an introduction to spike coding [41]. The first, simple code to have been proposed was the rate (or frequency) code, which simply observed that the rate of spiking discharge increases with stimulus intensity [42]. The rate code postulates that information is transmitted by the rate of spiking. In practice, the variable is the number of spikes within a time window, normalized by the duration of the window: rate $=n_{\text {spike }} / \Delta t$ (or equivalently, the variable $X_{i}$ can take $N_{i}$ values of rate). It is possible to consider variations of the spike rate using several consecutive time windows, each giving a variable $X_{i}$ and altogether forming a stochastic process. Temporal (or time or latency [43,44]) coding postulates that information is transmitted by the precise time of a spike. It corresponds to an instantaneous rate code, e.g. the limit of the rate code when the duration of the window tends to be small $\lim _{\Delta t \rightarrow 0}$ rate. There have been debates on whether nervous systems use spike time or rate coding, together with studies of information as a function of the duration of the window-bin $[41,45]$. Results of experiments show that the nervous system uses a temporal or rate code depending on the stimulus or task; simple stimuli with low information content or relevance evoke rate codes while highly informative, complex time-varying stimuli (for instance with high cognitive content), like natural conditions or stimulus the system has learned, tend to evoke a temporally precise spiking code [46-50]. Roughly, going from rate to temporal code increases information transmission rates, and in terms of consciousness the qualitative flow $[49,50,3]$. However, information transmission in neurons is not all-or-nothing: Vm transmits high information rates as unraveled by time-frequency wavelet decomposition (or time-energy in physic, that quantifies time vs frequency code $[49,50]$ ). Spike waveform and threshold vary significantly and further modulate synaptic transmission in an important part, if not all neurons. As reviewed [22,51,52] and investigated in [53-55], effective information transmission in real nervous systems is not a binary process and the entire membrane voltage codes. We consider that the electrical message and its structure are the code itself. Turning away from the unrealistic assumption that the code is sustained by ideal impulsional spikes (i.e. binary code) leads to the consideration of the more general electromagnetic "Vm code", which includes spiking events. What reads the neural code are the structures that effectively receive the signal-responses of the system, that is both ideal and physical homonculus [56,57]. Nervous systems activity corresponds to informational (co)chains of "ideal" and "physical" observers and emitters coding and decoding information, a multiplicity of information processing chains. Such realistic "analog" coding goes hand in hand with an obvious increase in the considered coding capacity of neural processes compared with digital approximation, an increase which is directly imposed by the increase of the size of the coding alphabet. In practice, studies of graded synaptic transmission [54] report high information transmission rates [58].

Consciousness is associative. The mainstream historical development of neuroscience has come to consider the nervous system as an associative dynamic memory. This central role of associativity is sustained in information cohomology by the fact that the algebra of random variables and conditioning is fundamentally associative, and that consciousness is the qualitative byproduct of the mnemonic activation and consolidation process. Hebb proposed the principle of associative plasticity and learning [59] generating cell assemblies and providing the physiological support of consciousness and memory.

Consciousness is (co)-relational cell assemblies. Following Hebb, the theory of neural assemblies was refined by Von der Malsburg [60] in his "correlation theory of brain function", proposing that the correlate of cognitionconsciousness lies in the patterns of neural activity quantified by correlations, and that simultaneously activated nerve cells represent the basic internal objects, further leading to the studies of synfire chain [61,62], and of synchrony and binding [63,64] including synchronous $40-100 \mathrm{~Hz}$ gamma oscillations during attentional states [65]. Following also [66-68], information cohomology, provide a natural ground to define cells assemblies and a statistically exact generalization of the "correlation theory", thanks notably to the remarkable status of mutual-informations.

Consciousness is the Gestalt. The theory of forms was developed by the school of Gestalt psychophysics [69-71], who provide elementary associative laws of binding that govern the construction of complex perceptual forms from basic elementary shapes. Moreover, Wertheimer and Kohler first proposed an isomorphism between mind and body, meaning that "objective brain processes (..) have functionally the same form and structure as subjective experiences" [72,73]. The principle of efficient coding, that the goal of sensory perception is to extract the redundancies and to find the most compressed representation of the data, was first stated by Attneave in 1954 [74] followed by Barlow [75]. Attneave notably claimed that any kind of symmetry and invariance are information redundancies and that Gestalt principles of perception can be defined in terms of information. Homology is the standard and appropriated 
mathematical theory to formalize and characterize what patterns or geometrical forms may be and to achieve the assimilation of symmetries and invariance as information quantities.

Consciousness is functional. The classical functional characterization of consciousness considers electrical activity as a function of stimulus. The process of consciousness is considered to be a function which consists in the "cross-correlation" or convolution of the stimulus with the neural response. Rather than a finalist view, it underlines the "dynamic" aspect of consciousness: a kind of relation between a domain and a codomain (that assigns to any element of the domain a single element of the codomain, creating an ordered pair). Function spaces provide very rich and diverse structures in mathematics, including Hilbert and Banach spaces, and are usually classified according to topological criteria. Information topology relies on an arguably general space of functions, the space of measurable functions, and provides a characterization of its structure. In biology, these input-output functions provide a "representation" or a coding of the (perceived) stimulus on a given functional basis. From the biophysical point of view, this function is usually characterized using the linear response theory, which studies the fluctuation and dissipation (i.e. the return to equilibrium) of a system following the external perturbation generated by the stimulus, as formalized by Kubo and applied to neural responses [76,77]. From an engineering perspective, this function is usually characterized using Volterra or Wiener's kernels methods [78-80] using white noise as input, what is sometime called the receptive field for sensory neurons [81-84] (but it has been characterized at all different organizational scales of the nervous system, cf. [3]).

Consciousness is probability-measure theory. The main theory and applied measure to formalize and quantify those assemblies is probability theory, e.g. Bayesian [85,86] and information theory. Following Kolmogorov [87], Jaynes [88], information cohomology [89,90] underlines that they are indeed a single inseparable theory. Bayesian theory and information theory provide the basic quantification of populational code. It consists in considering the multivariate case where each neuron corresponds to a variable or considering more complex time-dependent generalizations [91], hierarchical families of probability distributions [92], which considers higher order statistics of cell assemblies. For example, Ma and colleagues developed probabilistic population codes [93] to infer the stimulus from a population activity. The information topology characterizes the structure of such populational code according to their multivariate statistical dependencies. It is a leitmotif in biological and neuronal studies to investigate the role of noise, whether it be an un-mastered or "spontaneous" source of variability, and to propose that such a non-deterministic source is responsible for phenomena like consciousness [94,95], or living principle, as in the work of Brown which looks for the "vital forces" in living material [96]. Many studies have been dedicated to the functional role of noise and have pointed out that noise is "far from being a nuisance" [97,98]. Some have formalized noise, for example using stochastic resonance or self-organized criticality formalisms [99]. Control theory and the conceptualization of a channel of communication in information theory has also made use of such an ad-hoc noise source [100], using the deterministic "0 noise" formalism as a reference. Intrinsic variability has a very important role in human cognition and consciousness, as it allows free-will to be conceivable. As Ruelle remarked in his course on deterministic dynamical systems, critical-bifurcation points, hyperbolic saddle points, are the only places where the choice is given and left possible (see [101] for review). Recurrent network formalization using statistical physics, pioneered notably by Hopfield networks, introduced a new view on ongoing activity and thermal noise, proposing that it corresponds to the autonomous generative capacity of consciousness, illustrated in the context of the Helmholtz machine as accounting for the wake-sleep sequence and dreams [102]. The probabilistic approach, as notably proposed by Ma and colleagues [93] and also in information topology (cf. 3), generalizes the noise approach by considering biological and neural computation to be intrinsically probabilistic: the deterministic case is no longer the reference but a peculiar limit subcase. In such a view, any component of a (possibly nervous) system corresponds to a random variable, can be considered as a signal and a noise source and can be both emitter and receiver. Hence, the probabilistic and informational view attributes not only consciousness but also free will to varying observables.

Consciousness is an active adaptive process. As repeatedly underlined in neuroscience and cognitive studies, that coined such principle "action-perception" or "active-sensing", action is a necessary component of consciousness [103-109]. This inseparability of action and perception is a hallmark of the adaptive and dynamic nature of consciousness, which is a perpetually adaptive process; we can go a step beyond the dynamical nature of consciousness. Consciousness is variational by essence - what is constant, we are not conscious of; we all have a blind spot corresponding to a missing photoreceptor on the retina occupying an important part of our visual field, but none of us have ever seen it. A variable or source without noise is deterministic and is the trivial constant " 0 " information of an informational structure (cf. section 3): information only sees what varies, so to speak. In a sense, such probabilistic studies 
describe the heterogeneous structures of constitutive ongoing activity and the relative variations of ongoing activity. Indeed, the information topology framework introduces a variational view of probability, which was also proposed by Friston [110]. Since the biophysical study of Laughlin and Srinivasan [111,112] and the theoretical work of Linsker, Hinton, Nadal, Parga, Bell and Sejnowsky, mutual information maximization or relative entropy minimization has provided the main theoretical formalization of adaptation and neural plasticity [113-118]. Mathematically, this inherent dynamic-active aspect of consciousness is implemented homologically by the (left)-action of conditioning, a probabilistic expression of Klein's definition of a geometry as the action of group on a space [119,120], and that allows us to retrieve information paths as automorphism and stochastic processes, a stochastic expression of dynamical systems (cf. section 3).

\section{Information topology synthesis: consciousness's complexes and thermodynamic}

Information structures. Random variables are partitions of the atomic probabilities of a finite probability space $(\Omega, \mathcal{B}, P)$. The operation of joint-variable $\left(X_{1}, X_{2}\right)$ is the less fine partition, which is finer than $X_{1}$ and $X_{2}$; the whole lattice of partitions $\Pi[121]$ hence corresponds to the lattice of joint-variables [122,89]. A general information structure is defined in usual set-theoretic terms as a triplet $(\Omega, \Pi, P)$, and hence covers all the possible equivalence classes on atomic probabilities. A more general and modern expression is given in category and topos theory, in $[89,90]$. The image law of the probability $P$ by the measurable function of the joint-variables $\left(X_{1}, \ldots, X_{k}\right)$ is noted $\left(X_{1}, \ldots, X_{k} ; P\right)$. The fact that the lattice is a partially ordered set (poset) endowed with a refinement relation is central; it means that there is an intrinsic hierarchy of informational structure, just as in the general model of physical cognition of Schoeller, Perlovsky, and Arseniev [123]. The other common interpretation of this poset hierarchical structure, probably equivalent to the previous one, is that the ordering of the lattice provides a multi-scale, coarse to fine analysis, and each rank of the lattice provides an information analysis at the corresponding organizational level, as already formalized and applied by Costa et al. [124,125], who called it multiscale entropy in the context of time series. Hence, such formalism can be applied in the context of multiscale systems such as illustrated in [3] (in theory), and the entropy necessarily increases as more and more variables join, e.g. while progressing in organizational scales.

Action: In this general information structure, we consider the real module of all measurable functions $F\left(X_{1}, \ldots\right.$, $\left.X_{k} ; P\right)$ and the conditional expectation-mean (corresponding to informational conditioning) as the action of a variable $Y$ on the module, noted:

$$
Y . F\left(X_{1}, \ldots, X_{k} ; P\right)=k \sum_{y \in \mathcal{Y}}^{N_{y}} p(y) . F\left(X_{1}, \ldots, X_{k} ; P / Y=y\right)
$$

where $P / Y=y$ denotes the conditioning of the probability by the event $Y=y$, such that the action corresponds to the usual definition of conditional entropy. Centrally, the action of conditioning is associative [89,90]. Notably, Vigneaux was able to generalize all the formalisms presented here to Tsallis entropies by considering a deformed action (integer powers of probability in the expectation) [90], also giving a straightforward extension to quantized information. The complexes of measurable functions of random variables $X^{k}=F\left(X_{1}, \ldots, X_{k} ; P\right)$ and the cochain complex $\left(X^{k}, \partial^{k}\right)$ are noted as:

$$
0 \rightarrow X^{0} \stackrel{\partial^{0}}{\longrightarrow} X^{1} \stackrel{\partial^{1}}{\longrightarrow} X^{2} \stackrel{\partial^{2}}{\longrightarrow} \ldots X^{k-1} \stackrel{\partial^{k-1}}{\longrightarrow} X^{k}
$$

, where $\partial^{k}$ is the coboundary with a left action proposed by Hochschild for associative structures and rings [126], for Galois cohomology [127], and for homological algebra (see Cartan et Eilenberg's work [128] and for non-homogeneous bar complex (see Maclane [129]) is noted as:

$$
\begin{aligned}
& \left(\partial^{k}\right) F\left(X_{1} ; \ldots ; X_{k+1} ; P\right) \\
& \quad=X_{1} . F\left(X_{2} ; \ldots ; X_{k+1} ; P\right)+\sum_{i=1}^{k}(-1)^{i} F\left(X_{1} ; \ldots ;\left(X_{i}, X_{i+1}\right) ; \ldots ; X_{k+1} ; P\right)+(-1)^{k+1} F\left(X_{1} ; \ldots ; X_{k} ; P\right)
\end{aligned}
$$

For the first degree $k=1$, the 1-coboundary is $\left(\partial^{1}\right) F\left(X_{1} ; X_{2}\right)=X_{1} . F\left(X_{2}\right)-F\left(X_{1}, X_{2}\right)+F\left(X_{1}\right)$ and the 1-cocycle condition $\left(\partial^{1}\right) F\left(X_{1} ; X_{2}\right)=0$ gives $F\left(X_{1}, X_{2}\right)=F\left(X_{1}\right)+X_{1} . F\left(X_{2}\right)$, which is the fundamental chain 
law of information. Following Kendall [130] and Lee [131], it is possible to deduce from this chain law the functional equation of information and to uniquely characterize Shannon entropy as the first class of cohomology, up to the arbitrary multiplicative constant $k[89,90]$. It constitutes the main theorem that founded information topology. It appears by direct computation in this cohomology that mutual informations with an odd number of variables are minus the coboundary of even degrees $\partial^{2 k} F=-I_{2 k+1}$. Obtaining even mutual informations is achieved by introducing a second coboundary with either trivial or symmetric action $[132,89,133]$, giving the even mutual-informations as minus the odd symmetric coboundaries $\partial_{*}^{2 k-1} F=-I_{2 k}$. The independence of two variables $\left(I_{2}=0\right)$ is then directly generalized to k-variables and gives the cocycles $I_{k}=0$ [134]. Hence, information cohomology quantifies statistical dependencies and the obstruction to factorization.

What is the interest of these mathematical tools for cognition? The uniqueness of the obtained functions implies, in the case of their classical finite probabilistic application to empirical data, that the information functions are not only "good" but also the only ones to quantify statistical dependences and independences in the multivariate case. The finite-discrete symmetries of permutation groups, which are the structural ambiguity and the (co)differentials arising from Galois's theory, are equivalent to uncertainties and shared information arising from the "mathematical theory of communication". To comment on such a surprising and important fact, mutual informations are indeed (co)differential functions, a purely continuous operation arising from a finite and discrete context. Hilbert noted in his work on infinity, "the first unreasonable impression given to us by natural phenomena and matter is that of continuity" [135]: while physics repeatedly proved that objectively the input of our senses is finite and discrete, our consciousness construct the impression of continuity [135]. As expressed by Poincare [136], the construction of our continuous perception from discrete data can be proposed to be a cohomological operation by nature (even explaining Weber-Fechner's law) that mutual informations naturally fulfill. This is an important contribution of Galois's theory, further pursued by Lie, Picard-Vessiot and others, that allows us to conceive of the notion of continuity and of derivation yet holding in the discrete world, extending the classical Newtonian view. The second point of interest lies in the fact that this cohomology can be expressed as a Topos on a probability site [89,90], which allows the establishing of a multivalued constructive logic [3] generalizing binary boolean logic. Such logic can provide a basis for a probabilistic, biological and cognitive logic in which the excluded third is not a theorem (independent). Such constructive logic could underline the idea that those beliefs classically considered as complementary opposite statements - dualism - may indeed refer to a diversity of beliefs - pluralism.

The third point of interest is that cohomology is the science of the forms (patterns) of spaces. Information topology hence provides a preliminary theory of the shapes of probabilistic structures on which it is possible to develop methods of pattern recognition-characterization for machine learning and the quantification of epigenetic landscapes for biological adaptive dynamics, following Waddington and Thom [137,138,133]. Concerning classification-recognition aspects, information structures can be considered as universal: as a partition is equivalent to an equivalence class all possible classification are represented in an information structure. For example, this lattice can be understood as an algebraic formalization of deep networks, that is, networks with hidden layers of neurons for which the rank (dimension given in what follows) in the lattice gives the rank of a hidden layer and the connections correspond to coface maps (roughly, elementary projections or dimension increase). The random variables formalize neurons that are intrinsically probabilistic and possibly multivalued, generalizing binary and deterministic neurons such as McCulloch and Pitts' formal neurons. Information topology allows us to quantify the structure of statistical interactions within a set of empirical data and to express these interactions in terms of statistical physics, machine learning and epigenetic dynamics [133]. The combinatorics of general variable complexes being governed by Bell's numbers, their effective computation on classical computers is illusory. To circumvent those computational hardness, we define the sub-case of the simplicial cohomology of information, with a complexity in $\mathcal{O}\left(2^{n}\right)$ that can be implemented, but that neglects some of the possible dependencies. The computational hardness of Human consciousness is hence considered here as the main restriction for current Artificial Intelligence to claim having achieved Human-like consciousness. Considering simple recognition tasks or game benchmarks as well defined Turing test (with few degrees of freedom, low dimensional), deep learning algorithms already overpassed human abilities [139,140]. These methods establish a topological version of the Boltzmann and Helmholtz machines in machine learning [115,102] with ordered layered, named the Poincaré-Shannon machine. Notably, it answers to the requests stated by Seth, Izhikevich, Reeke, and Edelman, "that characterizing the relevant complexity of such a system will require a multidimensional analysis[...] qualia space is a high-dimensional space in which the axes reflect dimensions on which phenomenally experienced conscious scenes are discriminated" [141]. They also give a topological and algebraic answer, already present in essence in the work 
of $\mathrm{Hu}$ [142], to the questions of information decomposition that have been the subject of numerous publications and data applications, for instance the proposal of a non-negative composition or "unique information" [143-146].

The main theorems, definitions and data analysis [147,134,133] establish the following results, here included with comments about their relevance regarding consciousness and neural processing theories.

The marginal information $I_{1}$ are generalized internal energies and the k-mutual information $I_{k}$ are the free energy contributions of the k-body interaction. The total correlation proposed by Watanabe and Studeny $[148,149]$ to quantify dependences, or the IIT to quantify consciousness [1], $G_{k}=\sum_{i=2}^{k}(-1)^{i} \sum_{I \subset[n] ; \operatorname{card}(I)=i} I_{i}\left(X_{I} ; P\right)$, is the total free energy. As a result illustrated in [134], $I_{k}$ values allows to distinguish subpopulation, much better than $G_{k}$ that moreover do not have a direct homological meaning. In agreement with IIT theory that assigns consciousness according to $G_{k}$ measure [5,150], a conclusion following [147,134], is that genetic expression participate to consciousness, to its slow component on epigenetic regulation timescales. It allows to refund the (semi-)classical definitions of internal energy as a special case for phase space variable and the usual isotherm relation of thermodynamics $[151,152,133]$ :

$$
H\left(X_{1}, \ldots, X_{n}\right)=\langle E\rangle-G=U-G
$$

The marginal component, the internal energy, corresponds to a self-interaction, a reflexive component of consciousness that completes the model of Tononi and Edelman. Such a formalism could hence account for both the reflexive and the qualitative aspects of consciousness, in agreement with the Leibniz's monadic hypothesis.

Information paths in the simplicial lattice are in bijection with symmetric group and stochastic processes, providing a trivial topological generalization of the second principle of thermodynamics, following from conditional entropy positivity [133]. These paths correspond to the automorphisms of the partition lattice. This theorem generalizes Cover's theorem for Markov chains [153] and allows one to conjecture the existence of a Noether theorem for stochastic processes and discrete symmetries, notably following Baez and Fong [154]. Such a theorem should be considered as the topological version of the first principle of thermodynamics. Information paths and landscape directly account for standard causal criteria, like Granger causality and Transfer entropy, that generalize the later to the non-Gaussian case [155] and defined by Schreiber as a pairwise conditional mutual information [156]. We propose, completing thermodynamically an idea of Bergson [157], that conditional information positivity relations, given by the entropic cone and Shanonian inequalities [158], to be the support of our subjective time, whereas conditional information negativity relations, given by non-Shanonian inequalities [158], to be the support of space-like consciousness (simultaneity). The longest paths to the minima (equilibrium points) form the complex of minima of free energy. This complex formalizes the principle of minimal free energy in topology in complicated cases where multiple local minima co-exist. This simplicial complex provides a generic and consensual definition of a complex system, thus generalizing complex (1-complex) networks to larger dimensions. The multiplicity of these minima (facets) defines and quantifies diversity. This complex is proposed to provide a thermodynamical and mathematical formalization of the complexes developed in integrated information theory $[150,159,1]$. The possible coexistence of several facets that define the complex may explain the apparently paradoxical unity and diversity of consciousness: a conscious experience, corresponding to one facet, does not forbid the existence of some other conscious experience possibly less or more complex (of a different dimension), and that may be estimated as an unconscious process by the first one. Cognitively, a facet shall be understood as a composite memory process, a classical analog of what Griffiths, Omnes, and Gell-Mann and Hartle, called the consistent histories [160-162]. The quantification of consciousness proposed by Tononi and Edelman corresponds, for phase space variables, to free energy, and appears to be in agreement with the free energy principle proposed by Friston as an explanation for embodied perception [110], and generalizes it to non-Markovian observed relations [134] and to multiple critical points. Indeed, the complex of minima of free energy can be understood as a topologically discrete and finite generalization of the free energy principle of Friston that can be applied in the multivariate case with heterogeneous variables. Information topology also agrees in principles with the model of "projective consciousness" of Rudrauff and colleagues [163]. We moreover propose to replace the "self-evident" axioms proposed in the work of Tononi and colleagues [159] by the axioms of measure and probability theory, ultimately in the constructive logic framework [3]. Such axiomatization may allow to pursue the "artificial" consciousness opus of Turing and Wiener in some more refined, modern and hopefully computationally efficient formalism. The concept of "networks of networks" [164] corresponds topologically to the hypercohomology provided by the double complex (complexes of complexes in a homological sense, or a derived functor). It hence may also account for the Dehaene-Changeux model, which involves global neuronal workspaces and which is a "meta neural network", a network of neural networks constructed with neural integrate-and-fire neurons, thalamo-cortical columns and long-range cortical area networks 
[94,9,10]. Moreover, the minima of the complex corresponds to critical points which can be considered to correspond to the consciousness transition of their model. The application to data and simple theoretical examples shows that the positive maxima of $I_{k}$ identify the variables that co-vary the most, which could be called covariant assemblies or modules in the neuronal context. We hence propose that such positive modules provide a statistically rooted definition of neural assemblies, generalizing correlation measures to the nonlinear cases [165]. The negative minima of $I_{k}$, commonly called synergistic interactions [166] or negentropy following Schrodinger [167], identify the variables that most segregate the population, and hence detect clusters corresponding to exclusive differential activity in subpopulations $[147,134,133]$. This negativity of Free Energy component is discussed in [137] in the perspective of physic, and provides a topological signature of condensation phenomenon corresponding to the clustering of data point. It refines the negentropy principle of Schrödinger, stating that living systems feed upon negentropy or free-energy, by showing that even free-energy can have some negative components. It is remarkable that the pattern identified by positive and negative information corresponds to the two fundamental dual tasks of psychophysics, e.g. binding and segmentation, respectively. Moreover, minima of mutual information correspond in examples, and conjecturally in general to links, like the Borromean links. For example, the minima of $I_{3}$ for three Bernoulli variables is -1 bit' the variables are independent in pairs but linked at 3 by a purely 3-dimensional effect, a purely emergent collective interaction. In conclusion, the original contribution of this model with the cited theories, is to underline the fact that the essential properties of consciousness rely on structure and shape, not a single function, a single number or scalar. Moreover, the formalism highlights the fact that conscious experience, and also biological structures in general, correspond to discrete symmetries, to local energy minima, and to dynamical stochastic process. Considering the fact that symmetry could be a mathematical definition of aesthetics, which is historically a canonical definition, the formalism also further joins the model of physical cognition and that of dynamic logic by Schoeller, Perlovsky and Arseniev [123]: a Galoisian theory of e-motives or e-motions, a theory of ambiguity, "between crystal and smoke" [168], order and disorder, uncertainty and certainties (shared uncertainties) of the self and its environmental constitutive interactions.

\section{Conclusion - the global ecological synthesis}

The most obvious conclusion of this work is that consciousness is a natural and physical phenomenon, in principle ubiquitous, revealing itself in many different forms, that our human, highly specialized consciousness can hardly understand, imagine or even conceive. This informational synthesis was proposed by the ecological theory of mind and biology inaugurated by Gibson [169] and later formulated clearly by Atick [170] in information and adaptive terms. It should be clear that a precise and unified description and account of complex phenomenon such as the consciousness we experience unavoidably requires the use of the big machinery of algebraic topology and category, and even challenges it. The most basic reason for this is that it contains in its very constitutive foundation the germs of diversity, which are lost when one adds very few supplementary axioms or considers more specialized theories.

\section{Declaration of competing interest}

The author declares no competing financial interests.

\section{Acknowledgements}

This work was funded by the European Research Council (ERC consolidator grant 616827 CanaloHmics to J.M.G.; supporting M.T. and P.B.) and Median Technologies, developed at Median Technologies and UNIS Inserm 1072 Université Aix-Marseille, and at Institut de Mathématiques de Jussieu - Paris Rive Gauche (IMJ-PRG), and thanks previously to supports and hostings since 2007 of Max Planck Institute for Mathematic in the Sciences (MPI-MIS) and Complex System Instititute Paris-Ile-de-France (ISC-PIF). This work is dedicated to Daniel Bennequin and addresses a deep and warm acknowledgement to the researchers who participated to its development: i) for the electrophysiological and psychophysical part: Frédéric Chavane, Yves Frégnac, Sebastien Georges, Manuel Levy, Jean Lorenceau, Olivier Marre, Cyril Monier, Marc Pananceau, Peggy Series, ii) for the gene expression and homeostasis part: JeanMarc Goaillard, Monica Tapia iii) for the topological part: Daniel Bennequin, Juan-Pablo Vigneaux iii) for their encouragement, support and help: Henri Atlan, Frédéric Barbaresco, Habib Bénali, Paul Bourgine, Andrea Brovelli, 
Jürgen Jost, Guillaume Marrelec, Ali Mohammad-Djafari, Jean-Pierre Nadal, Jean Petitot, Alessandro Sarti, Jonathan Touboul. An extended version of this work has been deposited in Arxiv [3].

\section{References}

[1] Tononi G, Edelman G. Consciousness and complexity. Science 1998;282:1846-51.

[2] Bennequin D. Remarks on invariance in the primary visual systems of mammals, neuromathematics of vision part. Lecture Notes in Morphogenesis. Springer; 2014. p. 243-333.

[3] Baudot. Elements of consciousness and cognition. biology, mathematic, physics and panpsychism: an information topology perspective. arXiv:1807.04520, 2018.

[4] Yartsev M. The emperor's new wardrobe: rebalancing diversity of animal models in neuroscience research. Science 2017;358(6362):466-9.

[5] Paulson S. The spiritual, reductionist consciousness of Christoph Koch: what the neuroscientist is discovering is both humbling and frightening him. Nautilus 2017.

[6] Nagel T. What is it like to be a bat?. Philos Rev 1974;4:435-50.

[7] Edelman G, Tononi G. A universe of consciousness. how matter becomes imagination. New York: Basic Books; 2000.

[8] Dehaene S, Changeux J. Reward-dependent learning in neuronal networks for planning and decision making. Prog Brain Res 2000.

[9] Dehaene S, Changeux J. Experimental and theoretical approaches to conscious processing. Neuron 2011;70(2):200-27.

[10] Dehaene S, Changeux J, Naccache L, Sackur J, Sergent C. Conscious, preconscious, and subliminal processing: a testable taxonomy. Trends Cogn Sci 2006;10(5):204-11.

[11] Sperry R. Cerebral organization and behavior: the split brain behaves in many respects like two separate brains, providing new research possibilities. Science 1961;133:1749-57.

[12] Gazzaniga M. The split brain in man. Sci Am 1967;217(2):24-9.

[13] Chalmers D. Facing up to the problem of consciousness. J Conscious Stud 1995;2(3):200-19.

[14] Leibniz G. Discours de metaphysique VI; 1686.

[15] Leibniz G. The monadology. Oxford: Opera Philos.; 1714. http://home.datacomm.ch/kerguelen/monadology/. English translation by Robert Latta 1898.

[16] Galvani L. De viribus electricitatis in motu musculari commentarius (commentaire sur l'effet de l'électricité sur le mouvement musculaire) Bononiae - Institutus Scientiarium; 1791. http://sciences.amisbnf.org/node/1102.

[17] Gazzaniga M. The new cognitive neurosciences. 2nd edition. MIT Press; 1998.

[18] Sarraf S, Sun J. Functional brain imaging: a comprehensive survey. arXiv:1602.02225v4, 2016.

[19] John E. A field theory of consciousness. Conscious Cogn 2001;10:184-213.

[20] Pockett S. The nature of consciousness: a hypothesis. Writers Club Press; 2000.

[21] McFadden J. Synchronous firing and its influence on the brain's magnetic field. J Conscious Stud 2002;9:23-50

[22] Juusola M, Robinson H, de Polavieja G. Coding with spike shapes and graded potentials in cortical networks. BioEssays 2007;29(2):178-87.

[23] Schummers J, Yu H, Sur M. Tuned responses of astrocytes and their influence on hemodynamic signals in the visual cortex. Science 2008;320:1638-43.

[24] Preat T, Isabel G. Molecular and system analysis of olfactory memory in drosophila. In: Byrne JH, editor. Learning and memory: a comprehensive reference. Elsevier; 2008.

[25] Soden M, Jones G, Sanford C, Chung A, Guler A, Chavkin C, et al. Disruption of dopamine neuron activity pattern regulation through selective expression of a human kcnn3 mutation. Neuron 2013;80(4):1010-6.

[26] Monod J, Wyman J, Changeux J. On the nature of allosteric transition: a plausible model. J Mol Biol 1965;12:88-118.

[27] Fuller D, Chen W, Adler M, Groisman A, Levine H, Rappel W, et al. External and internal constraints on eukaryotic chemotaxis. Proc Natl Acad Sci USA 2010;107(21):9656-9.

[28] Davies E. Action potentials as multifunctional signals in plants: a unifying hypothesis to explain apparently disparate wound responses. Plant Cell Environ 1987;10(8):623-31.

[29] Holmes R, Christensen B, Wang R, Kwiat P. Testing the limits of human vision with single photons. In: Frontiers in optics. OSA Technical Digest; 2015.

[30] Tinsley J, Molodtsov M, Prevedel R, Wartmann D, Espigule-Pons J, Lauwers M, et al. Direct detection of a single photon by humans. Nat Commun 2016;7(1217):1-9.

[31] Bialek W, Schweitzer A. Quantum noise and the threshold of hearing. Phys Rev Lett 1985;54(7):725-8.

[32] Roy S, Llinas R. Relevance of quantum mechanics on some aspects of ion channel function. C R Biol 2009;332(6):517-22.

[33] Bohr N. Atomic physics and human knowledge. Chapman \& Hall; 1958.

[34] Hameroff S, Penrose R. Conscious events as orchestrated space-time selections. J Conscious Stud 1996;3(1):36-53.

[35] Penrose R. The emperor's new mind: concerning computers, minds and the laws of physics. Oxford University Press; 1989.

[36] Fusi S, Drew P, Abbott L. Cascade models of synaptically stored memories. Neuron 2005;45(4):599-611.

[37] Kadanoff L. Scaling laws for Ising models near tc. Physics 1966;2(6):263-72.

[38] Dyson F. The radiation theories of Tomonaga, Schwinger, and Feynman. Phys Rev 1949;75(3):486-502.

[39] Shirkov D. Evolution of the Bogoliubov renormalization group. arXiv:hep-th/9909024, 1999.

[40] Huang K. A critical history of renormalization. Int J Mod Phys A 2013;28(29):1-27.

[41] Rieke F. Spikes exploring the neural code, a Bradford book. Reprint edition. Computational neuroscience; 1999.

[42] Adrian E, Zotterman Y. The impulses produced by sensory nerve endings, part II: the response of a single end organ. J Physiol 1926;61:151-71. 
[43] Thorpe S, Fize D, Marlot C. Speed of processing in the human visual system. Nature 1996;382:520-2.

[44] Gawne T, Kjaer T, Richmond B. Latency: another potential code for feature binding in striate cortex. J Neurophysiol 1996;76(2):1356-60.

[45] Strong S, de Ruyter van Steveninck R, Bialek W, Koberle R. On the application of information theory to neural spike trains. Pac Symp Biocomput 1998:621-32.

[46] Bialek W, Rieke F, de Ruyter van Steveninck R, Warland D. Reading a neural code. Science 1991;252(5014):1854-7.

[47] Victor J, Purpura K. Nature and precision of temporal coding in visual cortex: a metric-space analysis. J Neurophysiol 1996;76(2):1310-26.

[48] Mechler F, Victor J, Purpura K, Shapley R. Robust temporal coding of contrast by v1 neurons for transient but not for steady-state stimuli. J Neurosci 1998;18:6583-98.

[49] Baudot P. Natural computation: much ado about nothing? An intracellular study of visual coding in natural condition. Master's thesis, Paris 6 university; 2006.

[50] Baudot P, Levy M, Marre O, Monier C, Pananceau M, Fregnac Y. Animation of natural scene by virtual eye-movements evokes high precision and low noise in v1 neurons. Front Neural Circuits 2013;7(206):1-29.

[51] Debanne D, Bialowas A, Rama S. What are the mechanisms for analogue and digital signalling in the brain?. Nat Rev Neurosci 2013;14:63-9.

[52] Zbili M, Rama S, Debanne D. Dynamic control of neurotransmitter release by presynaptic potential. Front Cell Neurosci 2016;10(278).

[53] Simmons P, de Ruyter van Steveninck R. Reliability of signal transfer at a tonically transmitting, graded potential synapse of the locust ocellar pathway. J Neurosci 2005;25(33):7529-37.

[54] de Ruyter van Steveninck R, Laughlin S. The rate of information transfer at graded-potential synapses. Nature 1996;379:642-5.

[55] Rama S, Zbili M, Bialowas A, Fronzaroli-Molinieres L, Ankri N, Carlier E, et al. Presynaptic hyperpolarization induces a fast analogue modulation of spike-evoked transmission mediated by axonal sodium channels. Nat Commun 2015;6:10163.

[56] Barlow H. Banishing the homonculus. In: Knill, Richards, editors. Perception as Bayesian inference. Cambridge University Press; 1995.

[57] Foldiack P. The 'ideal homunculus': statistical inference from neural population responses. Comput Neural Syst 1993:55-60.

[58] Borst A, Theunissen F. Information theory and neural coding. Nat Neurosci 1999;2:947-57.

[59] Hebb D. The organization of behaviour. New York: Wiley; 1949.

[60] Von der Malsburg C. The correlation theory of brain function. Internal Report 81-2, Germany: Dept. of Neurobiology, Max Planck Institute for Biophysical Chemistry, 3400 Gottingen; 1981.

[61] Abeles M. Local cortical circuits: an electrophysiological study. Berlin: Springer; 1982.

[62] Diesmann M, Gewaltig M, Aertsen A. Stable propagation of synchronous spiking in cortical neural networks. Nature 1999;402:529-33.

[63] Singer W, Gray C. Visual feature integration and the temporal correlation hypothesis. Annu Rev Neurosci 1995;18:555-86.

[64] Engel A, Fries P, Koenig P, Brecht M, Singer W. Temporal binding, binocular rivalry, and consciousness. Conscious Cogn 1999;8(2):128-51.

[65] Gold I. Does 40-Hz oscillation play a role in visual consciousness?. Conscious Cogn 1999;8(2):186-95.

[66] Curto C, Itskov V. Cell groups reveal structure of stimulus space. PLoS Comput Biol 2008;4(10).

[67] Singh G, Memoli F, Ishkhanov T, Sapiro G, Carlsson G, Ringach D. Topological analysis of population activity in visual cortex. J Vis 2008;8(11):1-18.

[68] Petri G, Expert P, Turkheimer F, Carhart-Harris R, Nutt D, Hellyer P, et al. Homological scaffolds of brain functional networks. J R Soc Interface 2014;6(11):101.

[69] Kohler W. Gestalt psychology: an introduction to new concepts in modern psychology. New York: Liveright; 1947.

[70] Wertheimer M. Gestalt theory. In: Ellis WD, editor. A source book of Gestalt psychology. London, England: Routledge \& Kegan Paul; 1924.

[71] Wertheimer M. Laws of organization in perceptual forms. In: Ellis WD, editor. A source book of Gestalt psychology. London, England: Routledge \& Kegan Paul; 1923.

[72] Wertheimer M. Isomorphism. Corsini Encyclopedia of Psychology 2010;1-2.

[73] Kohler W. Die physischen gestalten in ruhe und im stationaren zustand, abridged trans. In: Ellis WD, editor. A source book of gestalt psychology. New York: The Humanities Press; 1920. p. 71-88. Original work published in 1920.

[74] Attneave F. Some informational aspects of visual perception. Psychol Rev 1954;61(3):183-94. http://www.dc.uba.ar/materias/incc/teoricas/ Attneave1954.pdf.

[75] Barlow H. Possible principles underlying the transformation of sensory messages. In: Rosenblith WA, editor. Sensory communication. Cambridge, MA: MIT Press; 1961. p. 217-34.

[76] Kubo R. The fluctuation-dissipation theorem. Rep Prog Phys 1966;29(1):255-84.

[77] Stevens C. Inferences about membrane properties from electrical noise measurements. Biophys J 1972;12(8):1028-47.

[78] Wiener N. Nonlinear problems in random theory. MIT Press/John Wiley \& sons; 1958.

[79] Palm G, Poggio T. The Volterra representation and the wiener expansion: validity and pitfalls. SIAM J Appl Math 1977;33(2):195-216.

[80] Palm G, Poggio T. Stochastic identification methods for nonlinear systems: an extension of wiener theory. SIAM J Appl Math 1978;34(3):524-34.

[81] Hubel D, Wiesel T. Receptive fields of single neurones in the cat's striate cortex. J Physiol 1959;148:574-91.

[82] Hubel D, Wiesel T. Receptive fields, binocular interaction and functional architecture in the cat's visual cortex. J Physiol 1962;160(1):106-54.

[83] DeAngelis G, Ohzawa I, Freeman R. Receptive-field dynamics in the central visual pathways. Trends Neurosci 1995;8(10):451-8.

[84] Fournier J, Monier C, Levy M, Marre O, Fregnac Y. Hidden complexity of synaptic receptive fields in cat v1. J Neurosci 2014;34(16):5515-28.

[85] Griffiths TL, Kemp C, Tenenbaum JB. Bayesian models of cognition. In: Sun Ron, editor. Cambridge handbook of computational cognitive modeling. Cambridge University Press; 2008.

[86] Friston K. The history of the future of the Bayesian brain. NeuroImage 2012;62(2):1230-3.

[87] Kolmogorov A. Combinatorial foundations of information theory and the calculus of probabilities. Russ Math Surv 1983;38:29.

[88] Jaynes E. Probability theory: the logic of science. Posthum ed. Cambridge University Press; 2003. 
[89] Baudot P, Bennequin D. The homological nature of entropy. Entropy 2015;17(5):3253-318.

[90] Vigneaux J. Topology of statistical systems. A cohomological approach to information theory. Ph.D. thesis, Paris 7 Diderot University; 2019.

[91] Martignon L, Deco G, Laskey K, Diamond M, Freiwald W, Vaadia E. Neural coding: higher-order temporal patterns in the neurostatistics of cell assemblies. Neural Comput 2000;12(11):2621-53.

[92] Amari S. Information geometry on hierarchy of probability distributions. IEEE Trans Inf Theory 2001;47(5):1701-11.

[93] Ma W, Beck J, Latham P, Pouget A. Bayesian inference with probabilistic population codes. Nat Neurosci 2006;9(11):1432-8.

[94] Dehaene S, Changeux J. Ongoing spontaneous activity controls access to consciousness: a neuronal model for inattentional blindness. PLoS Biol 2005;3(5).

[95] Wyart V, Sergent C. The phase of ongoing eeg oscillations uncovers the fine temporal structure of conscious perception. J Neurosci 2009;29(41):12839-41.

[96] Brown R. A brief account of microscopical observations made in the months of June, July and August 1827 on the particles contained in the pollen of plants and on the general existence of active molecules. Massachusetts: Harvard University Press; 1828. Privately published In: Magie WF, editor. Source book in physics. Science Museum London; 1965.

[97] Vilardi A. The role of noise in brain dynamics processes. PhD Thesis, University of Trento, CIMeC, Centre for Mind/Brain Sciences; 2009.

[98] Eldar A, Elowitz M. Functional roles for noise in genetic circuits. Nature 2014;467(7312):167-73.

[99] Bak P, Tang C, Wiesenfeld K. Self-organized criticality: an explanation of 1/f noise. Phys Rev Lett 1987;59(4):381-4

[100] Shannon CE. A mathematical theory of communication. Bell Syst Tech J 1948;27:379-423.

[101] Baji V, Tan T. Information processing and living systems. Imperial College Press; 2005.

[102] Dayan P, Hinton G, Neal R, Zemel R. The Helmholtz machine. Neural Comput 1995;7:889-904.

[103] Merleau-Ponty M. Phenomenologie de la perception. Gallimard, coll. Tel; 1945.

[104] Llinas R. I of the Vortex. From neurons to self. A Bradford book. The MIT Press; 2002.

[105] Berthoz A. Physiologie de la perception et de l'action. Cours du collége de France 2000. https://www.college-de-france.fr/media/alainberthoz/UPL251487120930306111_AN_98_berthoz_2.pdf.

[106] Berthoz A. La decision. O. Jacob; 2003.

[107] Jeannerod M. Motor cognition: what actions tell to the self. 1st edition. Psychology series. Oxford University Press; 2006.

[108] Saraf-Sinik I, Assa E, Ahissar E. Motion makes sense: an adaptive motor-sensory strategy underlies the perception of object location in rats. J Neurosci 2015;35(23):8777-89.

[109] O'Regan J, Noe A. A sensorimotor account of vision and visual consciousness. Behav Brain Sci 2001;24:939-73.

[110] Friston K, Kilner J, Harrison L. A free energy principle for the brain. J Physiol (Paris) 2006;100(1-3):70-87.

[111] Srinivasan M, Laughlin S, Dubs A. Predictive coding, a fresh view of inhibition in the retina. Proc R Soc Lond 1981;216(1205):427-59.

[112] Laughlin S. The role of sensory adaptation in the retina. J Exp Biol 1989;146(39-62).

[113] Linsker R. From basic network principles to neural architecture. Proc Natl Acad Sci USA 1986;83:7508-12.

[114] Linsker R. Self-organization in a perceptual network. Computer 1988;21(3):105-17.

[115] Ackley D, Hinton G, Sejnowski TJ. A learning algorithm for Boltzmann machines. Cogn Sci 1985;9(1):147-69.

[116] Nadal N, Parga J-P. Sensory coding: information maximization and redundancy reduction, Neural information processing. World scientific series in mathematical biology and medicine, vol. 7In: Burdet G, Combe P, Parodi O, editors. 1999. p. 164-71.

[117] Nadal N, Parga J-P. Nonlinear neurons in the low noise limit: a factorial code maximizes information transfer. Netw Comput Neural Syst 1994;5:565-81.

[118] Bell T, Sejnowski AJ. An information maximisation approach to blind separation and blind deconvolution. Neural Comput 1995; 7(6):1129-59.

[119] Klein F. Vergleichende bertrachtungen uber neuere geometrische forschungen erlangen. Math Ann 1872;1893(1):460-97.

[120] Birkhoff G, Bennett M. Felix Klein and his "erlanger programm”. In: History and philosophy of modern mathematics. Univ. of Minnesota Press; 1988. p. 145-76.

[121] Andrews G. The theory of partitions. Cambridge: Cambridge University Press; 1998.

[122] Fresse B. Koszul duality of operads and homology of partition posets. Contemp Math Amer Math Soc 2004;346:115-215.

[123] Schoeller F, Perlovsky L, Arseniev D. Physics of mind: experimental confirmations of theoretical predictions. Phys Life Rev 2018. https:// doi.org/10.1016/j.plrev.2017.11.021.

[124] Costa M, Goldberger A, Peng C. Multiscale entropy to distinguish physiologic and synthetic rr time series. Comput Cardiol 2002;29:137-40.

[125] Costa M, Goldberger A, Peng C. Multiscale entropy analysis of biological signals. Phys Rev E 2005;71:021906.

[126] Hochschild G. On the cohomology groups of an associative algebra. Ann Math (2) 1945;46:58-67.

[127] Tate J. Galois cohomology, online course. http://wstein.org/edu/2010/582e/refs/, 1991.

[128] Cartan H, Eilenberg S. Homological algebra. Princeton: The Princeton University Press; 1956.

[129] Mac Lane S. Homology. Classic in Mathematics. Springer; 1975.

[130] Kendall D. Functional equations in information theory. Z Wahrscheinlichkeitstheor Verw Geb 1964;2:225-9.

[131] Lee P. On the axioms of information theory. Ann Math Stat 1964;35(1):415-8.

[132] Gerstenhaber M, Schack S. A hodge-type decomposition for commutative algebra cohomology. J Pure Appl Algebra 1987;48(1-2):229-47.

[133] Baudot P. The Poincare-Shannon machine: statistical physics and machine learning aspects of information cohomology. Entropy 2019;21(9):881.

[134] Baudot P, Tapia M, Bennequin D, Goaillard J. Topological information data analysis. Entropy 2019;21(9):869.

[135] Hilbert D. Sur l'infini. Hilbert's lectures on the infinite. Traduit par Andre Weil Paris; 1926. Edited in David Hilbert's Lectures on the Foundations of Arithmetic and Logic 1917-1933. Springer; 1924.

[136] Poincare H. Science and hypothesis. London: W. Scott; 1902. https://archive.org/details/scienceandhypoth00poinuoft. 
[137] Baudot P, Tapia M, Goaillard J. Topological information data analysis: Poincare-Shannon machine and statistical physic of finite heterogeneous systems. Preprint 2018040157, https://doi.org/10.20944/preprints201804.0157.v1, 2018.

[138] Tapia M, Baudot P, Dufour M, Formizano-Treziny C, Temporal S, Lasserre M, et al. Information topology of gene expression profile in dopaminergic neurons. 2017. BioArXiv168740.

[139] Silver D, et al. Mastering the game of go without human knowledge. Nature 2017;550(7676):354-9.

[140] Mnih V, Kavukcuoglu K, Silver D. Human-level control through deep reinforcement learning. Nature 2015;518(7540):529-33.

[141] Seth A, Izhikevich E, Reeke G, Edelman G. Theories and measures of consciousness: an extended framework. Proc Natl Acad Sci USA 2006;103(28):10799-804.

[142] Hu KT. On the amount of information. Theory Probab Appl 1962;7(4):439-47.

[143] Williams P, Beer R. Nonnegative decomposition of multivariate information. arXiv:1004.2515v1, 2010.

[144] Olbrich E, Bertschinger N, Rauh J. Information decomposition and synergy. Entropy 2015;17(5):3501-17.

[145] Bertschinger N, Rauh J, Olbrich E, Jost J, Ay N. Quantifying unique information. Entropy 2014;16:2161-83.

[146] Griffith V, Koch C. Quantifying synergistic mutual information. In: Prokopenko M, editor. Guided self-organization: inception. Berlin/Heidelberg: Springer; 2014. p. 159-90.

[147] Tapia M, Baudot P, Formizano-Treziny C, Dufour M, Temporal S, Lasserre M, et al. Neurotransmitter identity and electrophysiological phenotype are genetically coupled in midbrain dopaminergic neurons. Nat Commun 2018. Submitted for publication.

[148] Watanabe S. Information theoretical analysis of multivariate correlation. IBM J Res Dev 1960;4:66-81.

[149] Studeny M, Vejnarova J. The multiinformation function as a tool for measuring stochastic dependence. In: Jordan MI, editor. Learning in graphical models. Cambridge: MIT Press; 1999. p. 261-96.

[150] Tononi G, Boly M, Massimini M, Koch C. Integrated information theory: from consciousness to its physical substrate. Nat Rev Neurosci $2016 ; 17(7)$

[151] Adami C, Cerf N. Prolegomena to a non-equilibrium quantum statistical mechanics. Chaos Solitons Fractals 1999;10(10):1637-50.

[152] Kapranov M. Thermodynamics and the moment map. arXiv:1108.3472, 2011.

[153] Cover T. Which processes satisfy the second law?. In: Halliwell JJ, Perez-Mercader J, Zurek WH, editors. Physical origins of time asymmetry; 1994. p. 98-107.

[154] Baez J, Fong B. A Noether theorem for Markov processes. J Math Phys 2013;54:013301.

[155] Barnett L, Barrett A. Granger causality and transfer entropy are equivalent for Gaussian variables. Phys Rev Lett 2009;103:238701.

[156] Schreiber T. Measuring information transfer. Phys Rev Lett 2000;85(2):461-4.

[157] Bergson H. Time and free will: an essay on the immediate data of consciousness. Montana: Kessinger Publishing Company; 1910. Translated by FL Pogson.

[158] Yeung R. Information theory and network coding. Springer; 2007.

[159] Oizumi M, Albantakis L, Tononi G. From the phenomenology to the mechanisms of consciousness: Integrated information theory 3.0. PLoS Comput Biol 2014;10(5).

[160] Griffiths R. Consistent histories and the interpretation of quantum mechanics. J Stat Phys 1984;35:219.

[161] Omnes R. Logical reformulation of quantum mechanics, I: foundations. J Stat Phys 1988;53:893-932.

[162] Gell-Mann M, Hartle J. Quantum mechanics in the light of quantum cosmology. In: Zurek WH, editor. Complexity, entropy and the physics of information. Redwood City, CA: Addison-Wesley; 1990. p. 425-58.

[163] Rudrauf D, Bennequin D, Granic I, Landini G, Friston K, Williford K. A mathematical model of embodied consciousness. J Theor Biol 2017:428:106-31.

[164] D'Agostino G, Scala A. Networks of networks: the last frontier of complexity. Understanding complex systems. Springer; 2014.

[165] Reshef D, Reshef Y, Finucane H, Grossman S, McVean G, Turnbaugh P, et al. Detecting novel associations in large data sets. Science 2011;334:1518.

[166] Brenner N, Strong S, Koberle R, Bialek W. Synergy in a neural code. Neural Comput 2000;12:1531-52.

[167] Schrodinger E. What is life?; 1944. Based on lectures delivered under the auspices of the Dublin Institute for Advanced Studies at Trinity College, Dublin, in February 1943.

[168] Atlan H. Entre le cristal et la fumee. Essai sur l'organisation du vivant. Seuil; 1979.

[169] Gibson J. The ecological approach to visual perception. Boston: Houghton Mifflin; 1979.

[170] Atick J. Could information theory provide an ecological theory of sensory processing. Netw Comput Neural Syst 1992;3:213-51. 